\title{
Acute Kidney Injury Following Star Fruit (Averrhoa carambola) Ingestion in a Previously Healthy Young Man
}

\author{
ABDUL MUMITH RUHAN, ${ }^{1}$ PARASH ULLAH, ${ }^{1}$ MD. MOYEEN UDDIN, ${ }^{1}$ M. M. JAHANGIR ALAM, ${ }^{1}$ MD. SHAFIQUL BARI, ${ }^{1}$ \\ FAZLE RABBI CHOWDHURY ${ }^{1,2}$
}

\begin{abstract}
:
Star fruit (Averrhoa carambola) is a commonly available and popular fruit in many tropical and subtropical countries. Although star fruit induced oxalate nephropathy in patients with pre-existing renal impairment is well documented, reports on its effect on those with normal renal function are infrequent. Hereby we report a case where a young man with previously normal renal function presented with AKI that was attributable to consumption of star fruit. This write up illustrates the importance of obtaining the patient's history with respect to ingestion of star fruit in case of sudden and unexplained development of renal impairment.
\end{abstract}

Keywords: Star fruit, AKI, Oxalate nephropathy.

\section{Introduction:}

Acute kidney injury (AKI) is a syndrome characterized by the rapid loss of the kidney's excretory function along with an abrupt decline in the glomerular filtration rate (GFR) leading to accumulation of end products of nitrogen metabolism (urea and creatinine), and is typically diagnosed by increased level of urea and creatinine or decreased urine output or both. Amongst the toxic causes of AKI oxalate is one, which is highly contained in star fruit specially in sour type. ${ }^{1,2}$ The carambola or star fruit belongs to the Oxalidaceae family, species Averrhoa carambola. ${ }^{1}$ Slices cut in cross-section have the form of a star. It is believed to have originated from Ceylon and Moluccas and is now popular in many tropical and subtropical countries. In Bangladesh, it is locally known as "Kamranga"; and is taken frequently. Star fruit induced toxicity, manifesting as AKI and neurological impairment in individuals with previous renal impairment is well documented. ${ }^{3,4}$ However, AKI developing in individuals

1. Department of Medicine, Sylhet MAG Osmani Medical College, Sylhet, Bangladesh.

2. Centre for Tropical Medicine and Global Health, Nuffield Department of Medicine, University of Oxford, Oxford, UK

Corresponding author: Dr. Fazle Rabbi Chowdhury, Peter Medawar Building for Pathogen Research, South Parks Road, University of Oxford, OX1 3SY. Phone: +44 01865281657 , Cell: +44 07392757139, e-mail: fazle.chowdhury@ndm.ox.ac.uk. with normal renal function is not very common. ${ }^{5}$ We report a case of star fruit induced AKI in a person with previously normal renal function. To the best of our knowledge this is the first case of star fruit induced AKI to be reported from Bangladesh.

\section{Case report:}

A 25-year-old normotensive, non-diabetic male farmer presented first to a sub-district hospital of Bangladesh with the complaints of nausea, vomiting and scanty micturition for three days. On past medical history the duty doctors did not notice any significant clue. Due to erratic vomiting, patient was moderately dehydrated and he was started an intra venous fluid. They also advised serum creatinine which was found $5.4 \mathrm{mg} / \mathrm{dL}$. The patient was then referred to our hospital. On examination his pulse was 96 beats/ minute, blood pressure $110 / 80 \mathrm{~mm}$ of $\mathrm{Hg}$ and respiratory rate 28 breaths per minute. There was no history of fever, sore throat, skin rash, joint symptoms, or history of taking any reno-toxic drug or any product containing ethylene glycol or methoxyflurane. No prior history of kidney disease or gastric surgery was found. On further query to his dietary history, he said that first he started to feel nauseated, 4-5 hours after taking sliced star fruit at home. At that time he returned home from work and took them in empty stomach. They were sour in taste. He did not reveal this information to the previous medical center as he thought unimportant.

On 7th day, serum creatinine and eGFR were $7.8 \mathrm{mg} / \mathrm{dL}$ 
and $11 \mathrm{ml} / \mathrm{min} / 1.73 \mathrm{~m}^{2}$ respectively. Ultrasound scan of kidneys showed increased cortical echogenicity. Complete blood count, random blood sugar, serum electrolytes and urinalysis were normal. Hypercalcinuria, hyperuricosuria and hypomagnesuria were also excluded as contributory factor.

The patient was diagnosed as star fruit induced AKI, and conservative treatment was continued. On subsequent days patient's urinary volume increased along with decline in serum creatinine (Figure 1,2). On day 17 , serum creatinine and eGFR returned to normal level. We offered renal biopsy to the patient with proper concealing but unfortunately he denied. But his past and presumptive history make it quite clear that, start fruit was the culprit. Two months after recovery, patient was followed up with serum creatinine and urinalysis which came normal.

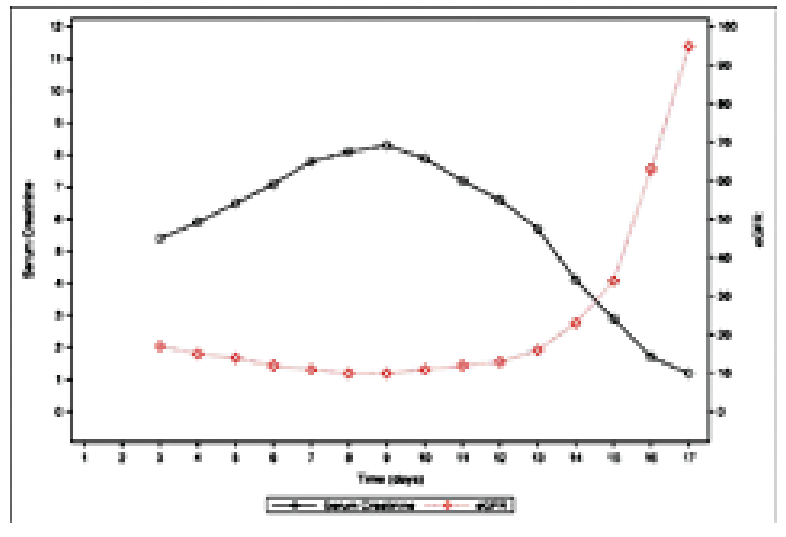

Figure 1: Trend of S. Creatinine and eGFR over time.

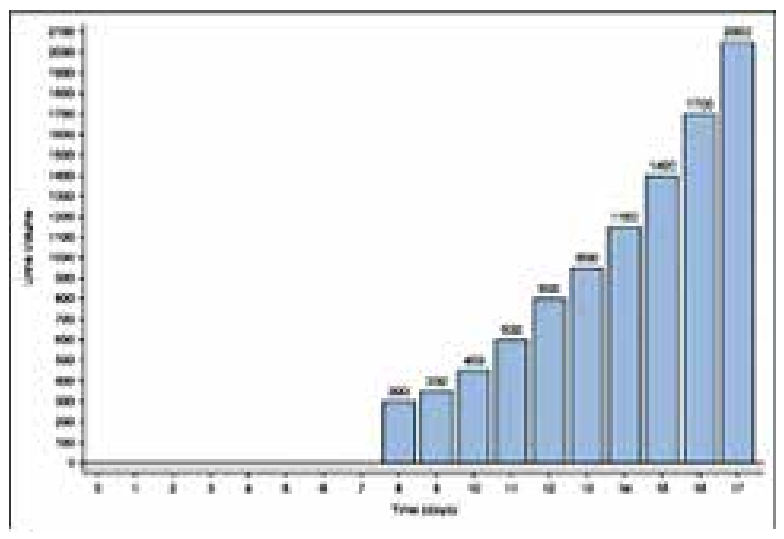

Figure 2: Improvement of urine output over time.

\section{Discussion:}

The first report describing star fruit induced oxalate nephrotoxicity in humans was published in 2001. ${ }^{1}$ The authors looked into two cases where the patients developed AKI after having star fruit juice; their kidney biopsies revealed histological findings consistent with acute oxalate nephropathy. Star fruit is a high source of oxalate. ${ }^{1,2}$ Fang $\mathrm{HC}$ et al. conducted a study on rats and found that excess star fruit consumption can cause oxalate nephropathy. ${ }^{6}$ Star fruits are of two types, sour and sweet. The sour type contains higher amount of oxalic acid. The lethal dose of soluble oxalate for human varies from 2 to 30 gram, and soluble oxalate concentration in star fruit varies from $80 \mathrm{mg} / 100 \mathrm{~mL}$ to $730 \mathrm{mg} / 100 \mathrm{~mL} .^{2,7}$ Our patient consumed around 700 grams star fruit.

Fang Hetal. described mechanisms of star fruit induced AKI, where he showed that nephrotoxicity leading to AKI is a result of both tubular obstruction by calcium oxalate crystals as well as apoptosis of the renal tubular epithelial cells. ${ }^{8}$ Risk factors for developing toxicity have been described as previously impaired renal function, ingestion of large amount of fruits or ingestion in an empty stomach and in a state of dehydration. ${ }^{1}$ Our patient also took it in empty stomach. Oxalic acid and its soluble salts are poisonous to humans and animals, whereas insoluble salts of calcium and magnesium oxalate are not. ${ }^{9}$ Oxalates ingested by humans may be precipitated by calcium or magnesium as an insoluble complex, which then is excreted in feces. ${ }^{9}$

The symptoms of star fruit induced nephrotoxicity are known to occur within hours of ingestion, and the most well documented ones are hiccups, nausea, vomiting, neuropsychiatric manifestations such as insomnia, seizure, mental confusion etc. ${ }^{3,5}$ Our patient also presented with nausea, vomiting along with scanty micturition within few hours. He took this on an empty stomach so that the protective effect of calcium and magnesium in food was lacking. In our case, although no previous diagnostic evidence was available, other features ensured us that the patient had no renal insufficiency of in the past. Star fruit toxicity, can cause neurotoxicity as well. In such cases, acute kidney injury prevents the excretion of caramboxin, a toxin found in star fruit, causing neurotoxic manifestations, all of which subside as soon as renal function is back to normal. ${ }^{5}$ In our case there was no neuropsychiatric manifestation. Majority of the patients require early renal replacement therapy while some can be managed 
conservatively. ${ }^{5,10}$ In our case clinical and biochemical improvement of the patient occurred gradually with conservative treatment only. Improvement of renal function was observed regularly, even 2 months after discharge from the hospital. Patients with previously normal renal function treated with or without dialysis had been reported to have good outcomes as happened to our patient. ${ }^{1,5}$

\section{Conclusion:}

We believe this form of AKI to be much more frequent than the cases been reported. We still do not know the actual magnitude of the problem in tropical and sub-tropical region. As more physicians become aware of such condition, we may observe an increased number of diagnosed cases. It is imperative that enquiries about star fruit ingestion should be a part of taking history in patients with unexplained deterioration of renal function. Yet we do not know the maximum recommended amount of star fruit or its juice beyond which toxicity would be likely to appear. It is necessary to determine the minimum amount of ingested star fruit that can provoke acute oxalate nephropathy.

Conflict of interest: None.

\section{References:}

1. Chen CL, Fang HC, Chou KJ, Wang JS, Chung HM. Acute oxalate nephropathy after ingestion of star fruit. Am J Kidney Dis. 2001;37:418-22.

2. Massey LK. Food oxalate: factors affecting measurement, biological variation, and bioavailability. J Am Diet Assoc. 2007;107:1191-4.

3. Wu CC, Denq JC, Tsai WS, Lin SH. Star fruit-induced neurotoxicity in two patients with chronic renal failure. J Med Sci. 2002;22(2):75-8.

4. Niticharoenpong K, Chalermsanyakorn P, Panvichian $\mathrm{R}$, Kitiyakara C. Acute deterioration of renal function induced by star fruit ingestion in a patient with chronic kidney disease. J Nephrol. 2006;19:682-6.

5. Neto MM, Silva GEB, Costa RS, Vieira Neto OM, Garcia-Cairasco N, Lopes NP, Haendchen PF, Silveira C, Mendes AR, Filho RR, Dantas M. Star fruit:simultaneous neurotoxic and nephrotoxic effects in people with previously normal renal function. NDT Plus. 2009;2(6):485-8.

6. Fang HC, Chen CL, Wang JS, Chou KJ, Chiou YS, Lee PT, Yeh JH, Yeh JH, Yeh MY, Chung HM. Acute oxalate nephropathy induced by star fruit in rats. Am J Kidney Dis. 2001;38:876-80.

7. Beier RC. Natural pesticides and bioactive components in foods. Rev Environ Contam Toxicol. 1990;113:47-137.

8. Fang H, Lee P, Lu PJ, Chen CL, Chang TY, Hsu CY, Chung HM, Chou KJ. Mechanisms of star fruit induced acute renal failure. Food Chem Toxicol. 2008;46(5):1744-52.

9. Sanz P, Reig R. Clinical and pathological findings in fatal plant oxalosis:A review. Am J Forensic Med Pathol. 1992;13:342-45.

10. Chen LL, Fang JT, Lin JL. Chronic renal disease patients with severe star fruit poisoning: hemoperfusion may be an effective alternative therapy. Clin Toxicol (Phila). 2005;43(3):197-9. 